\title{
Spatial Filter Design for Observation Spillover Suppression*
}

\author{
Yuichi MATSUMURA** and Naoki HOSOYA*** \\ ** Department of Mechanical Systems Engineering, University of Shiga Prefecture \\ 2500 Hassaka-cho, Hikone-shi, Shiga 522-8533, Japan \\ E-mail: matsu@mech.usp.ac.jp \\ *** Department of Production Sciences, Saitama University \\ 255 Shimo-Okubo, Sakura-ku, Saitama-shi, Saitama 338-8570, Japan \\ E-mail: hosoya@mech.saitama-u.ac.jp
}

\begin{abstract}
The present paper examines a method for the design of a spatial filter for suppressing modal observation spillover. A type of modal filter was proposed for eliminating several selected modal amplitudes from structural vibration responses. This filtering methodology can be realized by a small number of sensors, relative to the number of dominant modes in the frequency range of interest. The proposed filter can be designed using sensor placement to realize that the two subspaces, which are respectively spanned by a spatial filter vector and the selected target modal vectors, are orthocomplementary. The observation modal spillover associated with these selected modes is then suppressed. The effectiveness of the proposed filter was verified numerically for a cantilever beam and simulation results demonstrated potential for suppressing spillover in vibration control and health monitoring.
\end{abstract}

Key words : Modal Analysis, Filter, Measurement, Modal Filter, Observability, Spillover

\section{Introduction}

The present paper considers design methods for a spatial filter that uses fewer structural point sensors to reduce the observation modal spillover. This is because the spatial vibration control for suppressing several selected modes using the low-order controller is advantageous for controlling the associated sound radiation from a complex structure ${ }^{(1)}$. This potential ability has been sought in many critical applications to ensure a quiet environment.

An effective and convenient method of decreasing the modal numbers to be included for a sensor output involves the installation of sensors for the nodal points of an uncontrolled mode. While this technique is easy to realize and is useful for preventing the observation spillover, it cannot be utilized to make multiple modes simultaneously unobservable.

Modal filtering for observing only selected target modes is effective for suppressing observation modal spillover ${ }^{(2)-(5)}$.However, responses need to be measured using a sensor array, because the modal filtering is based on the orthogonality of the modal vectors, which is globally measured on a structure. Therefore, a number of issues related to the realization of modal filtering are known. For example, the number of modes in the target frequency range should be less than the number of sensors, and all modal vectors should be identified precisely in order to reduce the influence of measurement error in the calculation of oblique projectors ${ }^{(6)}$.

The present paper is concerned with the design methods of the point-sensor-based spatial filter, which causes any selected multiple modes to be unobservable. By designing the filter, which is orthogonal for only the mode of interest, the number of sensors required for this technique is drastically reduced compared to the conventional modal filtering techniques. Moreover, this spatial filter can be designed using only the modal vectors of the target modes, thus facilitating modal parameter identification. The technique is demonstrated numerically for a cantilever beam, principally for a scenario in which the number of sensors is two. 


\section{Conventional Method for Observation Modal Spillover Suppression}

\subsection{Sensor Outputs}

Point sensor outputs may be represented in the form of a modal model. Assuming the responses are measured at $N$ degrees of freedom and are sampled at $1 / \tau$ [Hz], the number of dominant modes is $Q$ in the frequency range of interest. Then, the sensor output $z\left(x_{n}, t_{k}\right)$ is given by the following equation for the proportional damping case

$$
z\left(x_{n}, t_{k}\right)=\sum_{q=1}^{Q} \phi_{q}\left(x_{n}\right) \eta_{q}\left(t_{k}\right),
$$

where $x_{n}$ is the sensor location of the $n$-th sensor, $t_{k}$ is equal to $k \tau, \phi_{q}\left(x_{n}\right)$ is the $n$-th element of the $q$-th modal vector $\phi_{q}$, and $\eta_{q}\left(t_{k}\right)$ is the modal coordinate responses of the $q$-th mode. The output vector $z\left(t_{k}\right)=\left[z\left(x_{1}, t_{k}\right) z\left(x_{2}, t_{k}\right) \cdots z\left(x_{N}, t_{k}\right)\right]^{T}$ of a sensor array can be written as

$$
\boldsymbol{z}\left(t_{k}\right)=\left[\begin{array}{cccc}
\phi_{1}\left(x_{1}\right) & \phi_{2}\left(x_{1}\right) & \cdots & \phi_{Q}\left(x_{1}\right) \\
\phi_{1}\left(x_{2}\right) & \phi_{2}\left(x_{2}\right) & \cdots & \phi_{Q}\left(x_{2}\right) \\
\vdots & \vdots & \ddots & \vdots \\
\phi_{1}\left(x_{N}\right) & \phi_{2}\left(x_{N}\right) & \cdots & \phi_{Q}\left(x_{N}\right)
\end{array}\right]\left[\begin{array}{c}
\eta_{1}\left(t_{k}\right) \\
\eta_{2}\left(t_{k}\right) \\
\vdots \\
\eta_{Q}\left(t_{k}\right)
\end{array}\right] \equiv \boldsymbol{\Phi} \boldsymbol{\eta}\left(t_{k}\right)
$$

where $\boldsymbol{\Phi}$ is a mode shape matrix, and $\boldsymbol{\eta}\left(t_{k}\right)$ is the column vector of the modal coordinate responses.

\subsection{Nodal Points Based Method}

Any mode can be unobservable by installing sensors on the nodal points of the mode. Assuming that the number of sensors is one, the target to be suppressed is the $s$-th mode. The sensor output on nodal point $x_{n_{s}}$ can be written using the following equation because $\phi_{s}\left(x_{n_{s}}\right)=0$ :

$$
z\left(x_{n_{s}}, t_{k}\right)=\sum_{q \neq s} \phi_{q}\left(x_{n_{s}}\right) \eta_{q}\left(t_{k}\right) .
$$

This technique is often used in structural vibration control, because it is easy to realize. However, this technique cannot be used to cause multiple modes to be unobservable, because, in general, the nodal lines of modes differ from one another.

\subsection{Modal Filter Based Method}

A modal filter is a coordinate transformation from physical to modal coordinates. Given that system responses can be uncoupled into modal coordinates, we can then observe modal component individually. Therefore, modal filters are used to render any selected modes unobservable in a filter output. For a case in which the number of sensors is larger than or equal to the number of dominant modes within the frequency range of interest, modal coordinate responses are calculated with the pseudo-inverse of a mode shape matrix,

$$
\boldsymbol{\eta}\left(t_{k}\right)=\boldsymbol{\Phi}^{\dagger} z\left(t_{k}\right)
$$

By using this equation, we can observe individual modal components. However, because it is based on an oblique projection theory ${ }^{(6)}$, this modal decomposition is sensitive to measurement error. The identification accuracy of modal vectors is, in general, inferior to that of natural frequencies, and the accuracy is often insufficient for oblique projection. Conventionally, the number of sensors employed is greater than twice the number of dominant modes within the frequency range of interest. Moreover, this technique requires complete and accurate estimates of all of modal vectors in order to compute faithful projectors; any errors in the estimation of modal vectors is propagated to all of the oblique projectors ${ }^{(6)}$. 


\section{Design Method of a Spatial Filter for Modal Spillover Suppression}

\subsection{Concept}

A spatial filter is introduced in order to make selected multiple modes unobservable. The concept of filter design is shown in Fig. 1. Note that the target modes to made unobservable are $s=\left[s_{1}, s_{2}, s_{3}\right]$. First, the case in which the number of sensors is two is described. Assuming that we are able to place the sensors in adequate sensor locations that satisfy the requirement for modal vectors to lie along the same straight line, we have

$$
\phi_{s_{1}}=\lambda_{1} \phi_{s_{2}}=\lambda_{2} \phi_{s_{3}},
$$

where $\lambda_{1}$ and $\lambda_{2}$ are any non-zero real numbers. Then, spatial filter $\psi_{s}$ can be defined as an orthogonal vector to $\phi_{s_{1}}$

$$
\psi_{S}^{T} \phi_{s_{1}}=0
$$

This spatial filter is then orthogonal to all of the target modes if Eq. (5) holds

$$
\boldsymbol{\psi}_{\boldsymbol{S}}^{T} \boldsymbol{\phi}_{s_{\ell}}=0, \quad \ell=1,2,3 .
$$

Assuming that, except for target modes, none of the modes are orthogonal to the spatial filter:

$$
\psi_{s}^{T} \phi_{q} \neq 0, \quad \forall q\left(\neq s_{1}, s_{2}, s_{3}\right),
$$

spatial filtering can be calculated as follows:

$$
\psi_{S}^{T} z\left(t_{k}\right)=\sum_{q \neq s_{1}, s_{2}, s_{3}} \psi_{S}^{T} \phi_{q} \eta_{q}\left(t_{k}\right)
$$

In this filtered time domain response, only the target modes $s=\left[s_{1}, s_{2}, s_{3}\right]$ are suppressed.

Next, a scenario in which the number of sensors is three is described. Here, sensors are located in optimal locations that satisfy the requirement for modal vectors to lie within the same plane. The proposed spatial filter $\psi_{s}$ can then be defined as the normal vector of the plane.

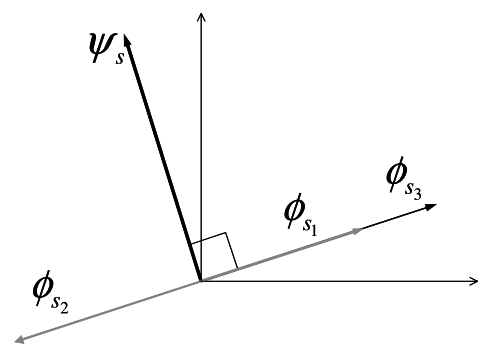

(a) Number of sensor: 2

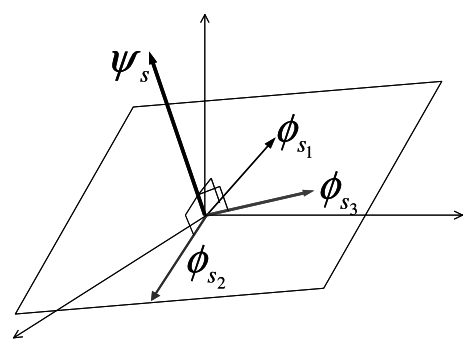

(b) Number of sensor: 3

Fig. 1 Concept of the proposed spatial filter 


\subsection{Generalization}

The concept shown in Fig. 1 is extended to the scenario in which the number of sensors is $N$. Suppose that the target modes to be made unobservable are $s_{1}, s_{2}, \cdots, s_{M}$, and the number of target modes is larger than or equal to the number of sensors. The proposed spatial filter may be designed as follows:

(1) Sensor placement is optimized to satisfy the dimension of the subspace $V$ spanned by modal vectors $\phi_{s_{\ell}}(\ell=1,2, \cdots, M)$ is $N-1$ :

$\operatorname{dim} V=N-1$.

( 2 ) Calculate the spatial filter $\psi_{S}$ that spans orthocomplementary subspace $W$ of subspace $V$ :

$$
E^{N}=V \dot{\oplus} W
$$

In this filter design, only estimates of target modes are required.

\subsection{Sensor Placement Based Design Method}

As an example, consider the case in which we use two sensors for suppressing two or three modes. In this case, the number of modes is larger than or equal to the number of sensors.

Case 1: Suppose we suppress two modes $s_{1}$ and $s_{2}$ with two point sensors. Sensor placement is optimized so that the modal vectors of the target modes are linearly dependent. We then define the following function in order to calculate the determinant of the mode shape matrix, the columns of which consist of target modes:

$$
W_{s_{1}, s_{2}}\left(x_{1}, x_{2}\right) \equiv \phi_{s_{1}}\left(x_{1}\right) \phi_{s_{2}}\left(x_{2}\right)-\phi_{s_{1}}\left(x_{2}\right) \phi_{s_{2}}\left(x_{1}\right) .
$$

Optimal sensor locations $x_{1}$ and $x_{2}$ are determined so as to satisfy the following conditions:

$$
W_{s_{1}, s_{2}}\left(x_{1}, x_{2}\right)=0, \quad x_{1} \neq x_{2} .
$$

Case 2: Suppose we suppress three modes $s_{1}, s_{2}$ and $s_{3}$ with two point sensors. In this case, sensor placement is performed in a manner similar to that described in Case 1. Only two conditions, defined below, are added to Eq. (13).

$$
\begin{aligned}
& W_{s_{1}, s_{3}}\left(x_{1}, x_{2}\right)=0 \\
& W_{s_{2}, s_{3}}\left(x_{1}, x_{2}\right)=0
\end{aligned}
$$

\subsection{Determination of Spatial Filter}

A spatial filter vector $\psi_{S}$ is calculated so that the filter vector is orthogonal to the target modes $\boldsymbol{\phi}_{s_{1}}$ and $\boldsymbol{\phi}_{s_{2}}$. These two vectors are linearly dependent on sensor placement, as described above, because the sensor placement was optimized so that these vectors are linearly dependent. Therefore, the spatial filter vector may be determined using the modal vector of the $s_{1}$-th mode, as follows:

$$
\psi_{s}=\left[\begin{array}{c}
\phi_{s_{1}}\left(x_{2}\right) \\
-\phi_{s_{1}}\left(x_{1}\right)
\end{array}\right] .
$$

\section{Intended Use}

After processing the proposed filter, we can obtain a one-dimensional time domain signal. In this section, we address the physical meaning and intended application of this signal, since the 1 st and 2 nd modes are simultaneously unobservable. The filtered signal can be expressed as

$$
\psi_{\boldsymbol{S}}^{T} z\left(t_{k}\right)=\sum_{q=3}^{Q} \psi_{\boldsymbol{S}}^{T} \phi_{q} \eta_{q}\left(t_{k}\right)
$$


The proposed filtering is the same as the modal filter for the case in which the number of dominant modes in sensor outputs is three $(Q=3)$. The modal coordinate of the 3 rd mode $\eta_{3}\left(t_{k}\right)$ can be directly calculated by Eq.(17) for this case,

$$
\psi_{s}^{T} z\left(t_{k}\right)=\psi_{s}^{T} \phi_{3} \eta_{3}\left(t_{k}\right)
$$

This coefficient $\boldsymbol{\psi}_{S}^{T} \boldsymbol{\phi}_{3}$ is known at the design stage. Then, modal coordinate $\eta_{3}\left(t_{k}\right)$ can be obtained by dividing Eq. (18) by a scalar coefficient $\psi_{S}^{T} \phi_{3}$,

$$
\eta_{3}\left(t_{k}\right)=\frac{\psi_{\mathbf{s}}^{T} z\left(t_{k}\right)}{\psi_{S}^{T} \phi_{3}} .
$$

This calculation is simply the modal filtering of the 3rd mode.

For the case in which the number of dominant modes is greater than four, we describe the physical meaning of the filtered signal. The coefficient $\boldsymbol{\psi}_{\boldsymbol{S}}^{T} \boldsymbol{\phi}_{q}$ of Eq. (17) is an element of the $q$-th modal vector $\phi_{q}\left(x_{p}\right)$ corresponding to a fictitious sensor location $x_{p}$,

$$
\phi_{q}\left(x_{p}\right) \equiv \psi_{S}^{T} \phi_{q}
$$

Then, the filtered time domain signal is treated as the sensor output $z\left(x_{p}, t_{k}\right)$ measured at the fictitious sensor location $x_{p}$, which is located at the nodal points of both the 1 st and 2 nd modes.

$$
z\left(x_{p}, t_{k}\right) \equiv \psi_{S}^{T} z\left(t_{k}\right)=\sum_{q=3}^{Q} \psi_{S}^{T} \phi_{q} \eta_{q}\left(t_{k}\right)
$$

This filtered signal is useful for eliminating neighboring modes to observe modes of interest in structural health monitoring ${ }^{(7)}$.

\section{Numerical Examples}

\subsection{Cantilever Beam}

Numerical analysis was performed using the cantilever beam shown in Fig. 2. The beam was consructed of SUS304 steel and had dimensions of $300 \times 30 \times 3 \mathrm{~mm}$. A finite element model with 601 nodes and 600 elements was developed in order to produce simulated experimental data. This mesh enabled us to install sensors on the beam at intervals of $0.5 \mathrm{~mm}$, with the locations of two sensors $x_{1}$ and $x_{2}$ satisfies the following condition:

$$
x_{1}<x_{2}
$$

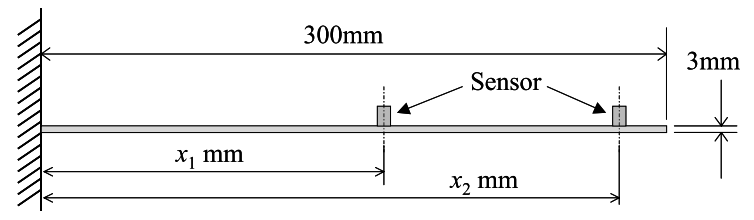

Fig. 2 Cantilever beam

Impulse responses obtained by forcing the free-edge of the beam were used as sensor outputs. The first four bending modes were used in this verification, and the modal properties of these modes are shown in Table 1.

\begin{tabular}{c|c|c|c}
\multicolumn{2}{c}{ Table 1 } & \multicolumn{2}{c}{ Modal property of the bending mode } \\
\hline Mode & Analytical & \multicolumn{2}{c}{ Simulated Experimental } \\
\cline { 2 - 4 } & $\Omega_{q}[\mathrm{~Hz}]$ & $\omega_{d q}[\mathrm{~Hz}]$ & $\zeta_{q}[\%]$ \\
\hline 1st & 26.72 & 26.72 & 0.084 \\
2nd & 167.5 & 167.5 & 0.526 \\
3rd & 468.9 & 468.9 & 1.473 \\
4th & 918.8 & 918.8 & 2.887 \\
\hline
\end{tabular}




\subsection{Two-mode Suppression}

A spatial filter capable of simultaneously suppressing any two modes was designed. The sensor placement that satisfied Eq. (13) is shown by solid circles in Fig. 3. The computation for determining the sensor placement was performed using the MATLAB function fzero.m by fixing the location of the first sensor $x_{1}$ at intervals of $0.5 \mathrm{~mm}$. Here, the initial value of the location of the second sensor $x_{2}$ was set on the free-edge of the beam.
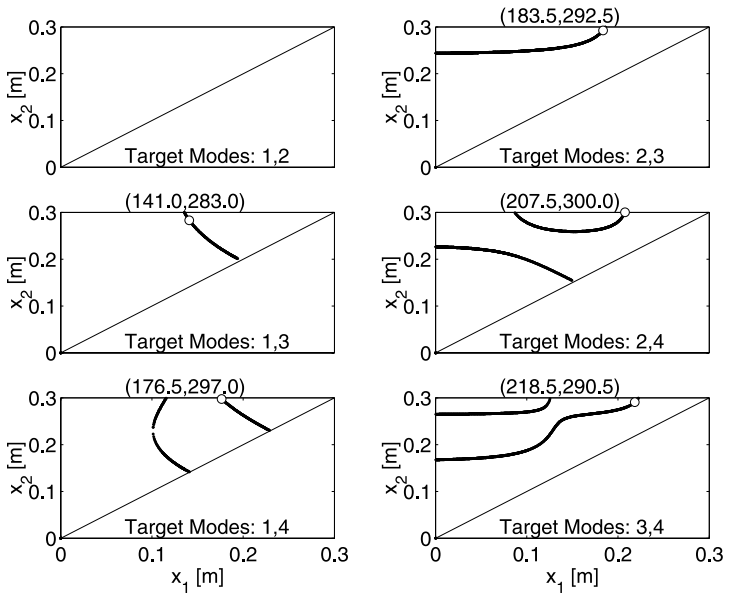

Fig. 3 Sensor placement for two-mode suppression using two sensors

Figure 3 shows that simultaneous suppression of the 1 st and 2 nd modes cannot be achieved, and that, for other mode combinations, the sensor placement for simultaneously suppressing two modes can be easily selected from a continuously distributed location. The value of the function $W_{1,2}\left(x_{1}, x_{2}\right)$ for sensor placement, as shown in Fig. 4, does not miss the zero-crossing point in the MATLAB function fzero.m. Figure 4 shows that there is no sensor placement that satisfies $W_{1,2}\left(x_{1}, x_{2}\right)=0$ under the condition $0<x_{1}<x_{2}<L$, where $L$ is the length of the beam.

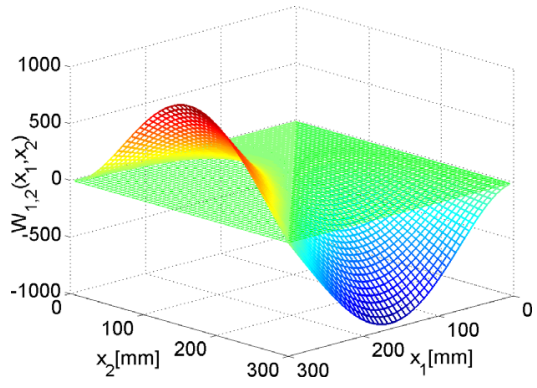

Fig. 4 Value of function $W_{1,2}\left(x_{1}, x_{2}\right)$

The open circles and the values above the open circles in Fig. 3 show the artificially selected quasi-optimum sensor placement under the condition that sensor location can be set at intervals of $0.5 \mathrm{~mm}$. Figure 5 shows modal vectors at the sensor placement, which are shown as open circles in Fig. 3, and the angle between these modal vectors. The modal vectors are almost linearly dependent after sensor placement. The spatial filter is then obtained such that it is orthogonal to the lowest $s_{1}$-th mode. Note that the $s_{2}$-th mode is not orthogonal to this spatial filter, because two modal vectors $\phi_{s_{1}}$ and $\phi_{S_{2}}$ are not rigorously linear dependent in practice.

$$
\begin{aligned}
& \psi_{S}^{T} \phi_{S_{1}}=0 \\
& \psi_{S}^{T} \phi_{S_{2}} \approx 0
\end{aligned}
$$



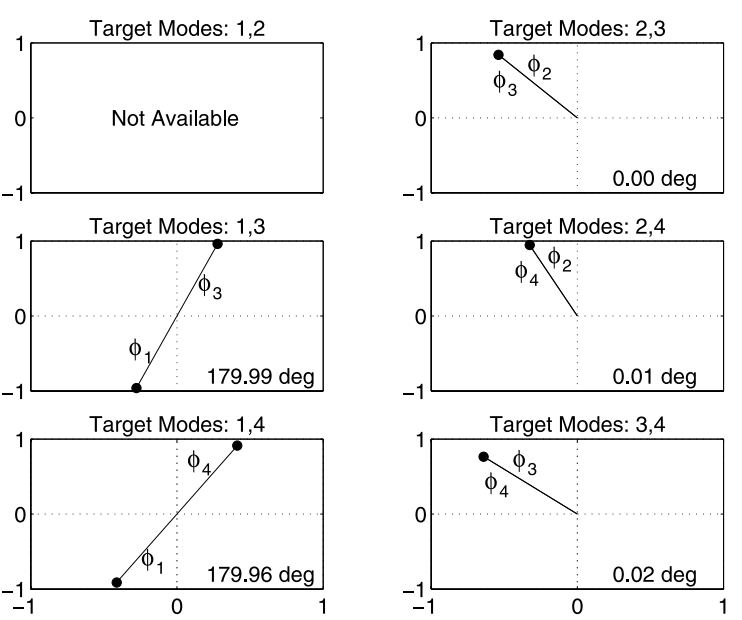

Fig. 5 Angle between simulated experimental modal vectors for the selected sensor location

Figure 6 shows the filtering results for the sensor outputs measured at the location shown by the open circle in Fig. 3. The left-hand column of Fig. 6 shows the weighting coefficients $\boldsymbol{\psi}_{S}^{T} \boldsymbol{\phi}_{q}(q=1,2,3,4)$ of modal coordinate responses. The center column of Fig. 6 shows the filtering results. The right-hand column of Fig. 6 shows the PSD of the filtered responses. In the left column of Fig. 6, the weighting coefficients of the $s_{2}$-th mode is approximately equal to zero, and so the weighting coefficients of the $s_{1}$-th mode is equal to zero. Therefore, the filtered responses shown in the center and right-hand columns of Fig. 6 consist of two residual modes. Two-mode suppression with two sensors can be realized by the proposed method for this case study.
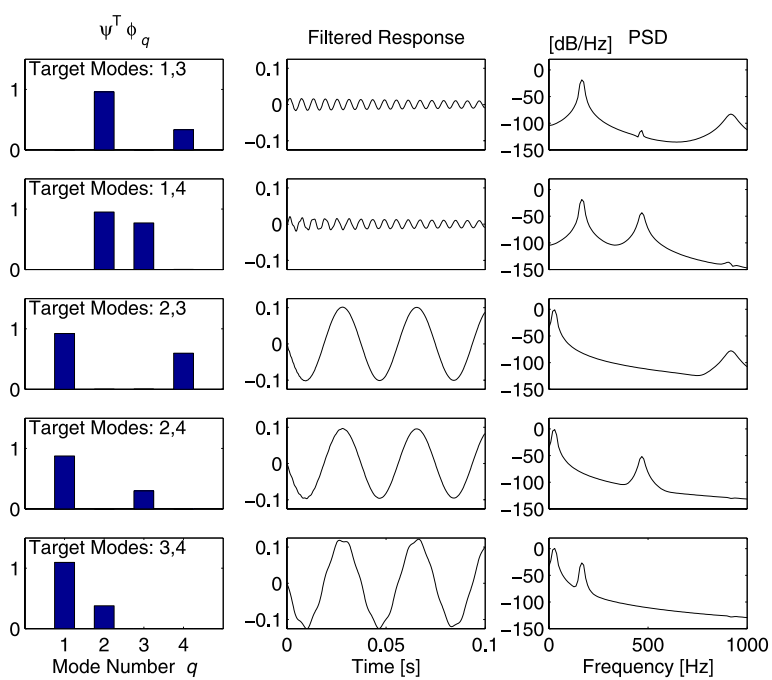

Fig. 6 Orthogonality checks and filtered results

\subsection{Three-mode Suppression}

A spatial filter that simultaneously suppresses any three modes was designed and is applied to the outputs of two sensors. Sensor placement that simultaneously satisfies Eqs. (13), (14), and (15) is obtained as an intersection point of the curves shown in Fig. 3.

The open circles denoted A to E in Fig. 7 show the respective intersection points. The sensor placement corresponding to these open circles is shown in Table 2. Moreover, the sensor location shown in Table 2 is indicated as mode shapes in Fig. 8. The positions indicated 

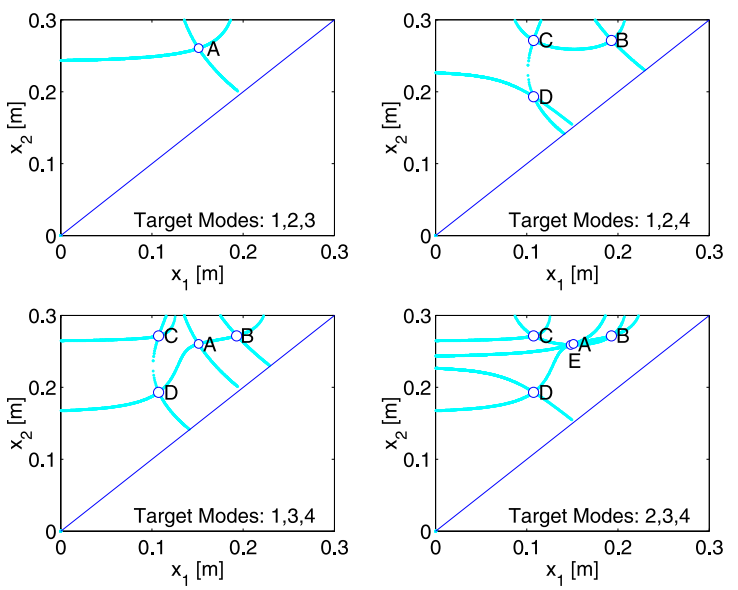

Fig. 7 Selection of sensor placement for three-mode suppression with two sensors

in Fig. 8 by letters separated by a comma, for example 'C,D', indicates the common sensor location of point $\mathrm{C}$ and point $\mathrm{D}$. In Fig. 8, sensor placements $\mathrm{A}$ through $\mathrm{D}$ correspond to the nodal points of a single mode. This indicates that the points A through D in Fig. 7 are the intersection points of two curves. Therefore, the sensor placement corresponding to points $\mathrm{A}$ through D is not the solution that simultaneously satisfies Eqs. (13), (14), and (15).

Table 2 Intersection point

\begin{tabular}{c|c|c|c}
\hline \multirow{2}{*}{ Point } & \multicolumn{2}{|c|}{ Sensor Placement } & Reference \\
\cline { 2 - 3 } & $x_{1}[\mathrm{~mm}]$ & $x_{2}[\mathrm{~mm}]$ & \\
\hline A & 151.0 & 260.5 & Nodal points of 3rd mode \\
B & 193.0 & 271.5 & Nodal points of 4th mode \\
C & 107.5 & 271.5 & Nodal points of 4th mode \\
D & 107.5 & 193.0 & Nodal points of 4th mode \\
E & 148.0 & 259.0 & Modal filtering of 1st mode \\
\hline
\end{tabular}
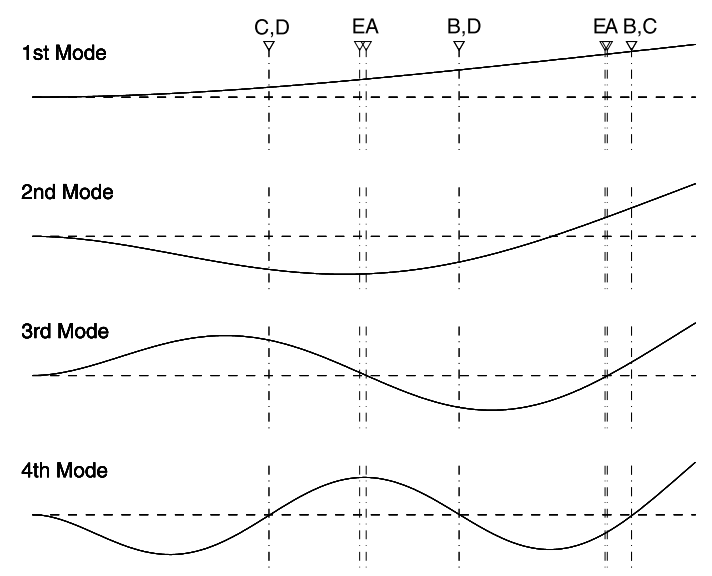

Fig. 8 Mode shapes and sensor placement candidates

Point E in Fig. 7 is the point at which three curves approach each other. Sensor placement corresponding to this point produces an approximately linearly dependent set of target modes, which is sufficient for designing the proposed spatial filter. This result indicates that modal filtering of first mode is realized in this case. The long dashed lines in Fig. 9 show the impulse responses and their PSDs, as measured at sensor placement corresponding to point E. The responses consist of four mode components. Figure 10 shows a filtered response and its PSD. These signals are scaled by the weighting coefficient $\boldsymbol{\psi}_{S}^{T} \boldsymbol{\phi}_{1}$, as shown in Eq. (9). In this figure, the filtered response consists of only a single mode, and the target modes 
are suppressed sufficiently. Moreover, the filtered response agrees well with the first mode components obtained by theoretical calculation. Premultiplying $\hat{\eta}_{1}\left(t_{k}\right)$ given in Fig. 10 by the modal vector of the first mode yields the first mode components at each sensor location. The estimated first mode components are shown by the solid lines in Fig. 9. Modal filtering can be realized by the proposed method if the number of sensors is less than the number of dominant modes within the frequency range of interest.
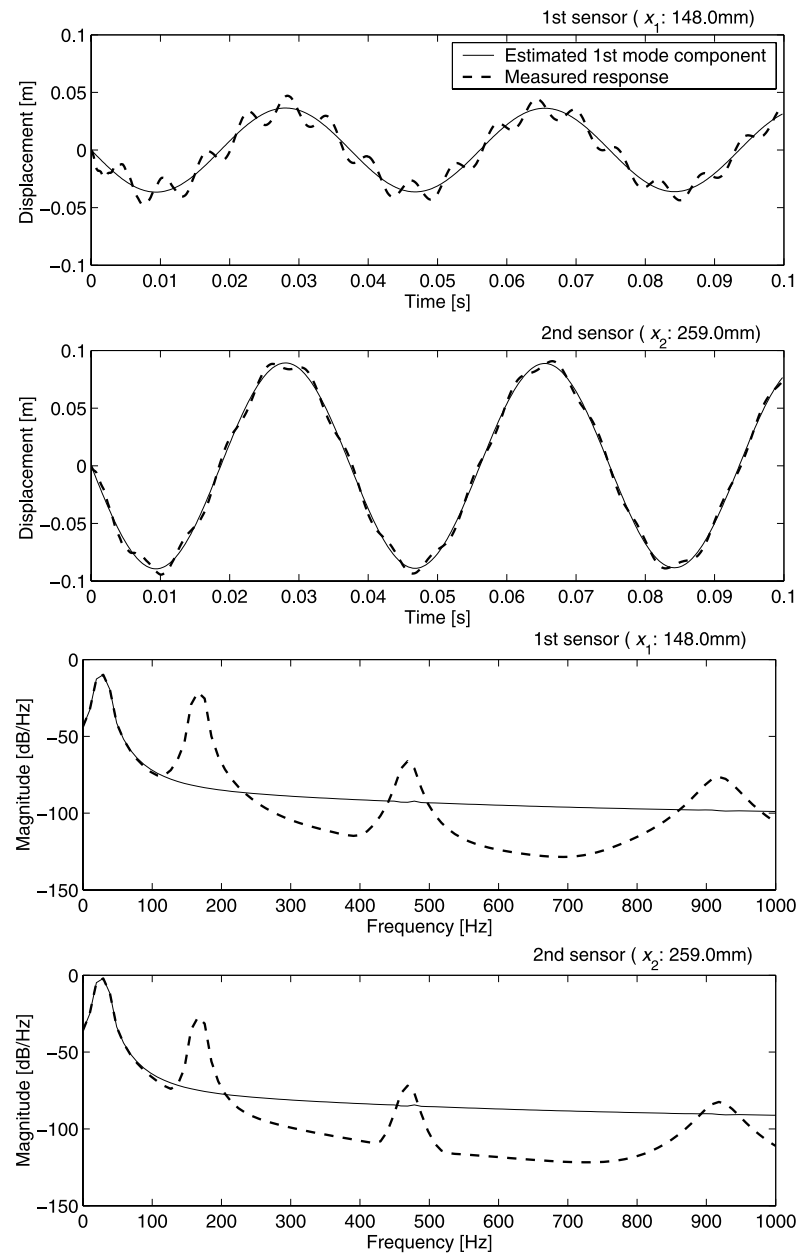

Fig. 9 Impulse responses and estimated first-mode components at the E-set sensor location

\section{Conclusion}

The design method of a spatial filter for suppressing modal observation spillover is proposed. This spatial filter is used for eliminating the several selected modal amplitudes from structural vibration responses. As an example of this technique, the spatial filter was designed for a cantilever beam and was applied to the responses measured by a few point sensors. Some major conclusions are listed as follows:

( 1 ) The proposed filtering methodology can be realized by a small number of sensors, relative to the number of dominant modes in the frequency range of interest.

( 2 ) Only the estimates of target modes are required in order to design the proposed filter. Therefore, the design method does not require complete and accurate estimates of all of the modal vectors.

( 3 ) Realization of the proposed technique was verified numerically for a cantilever beam, and three-mode suppression with two point sensors was realized. 

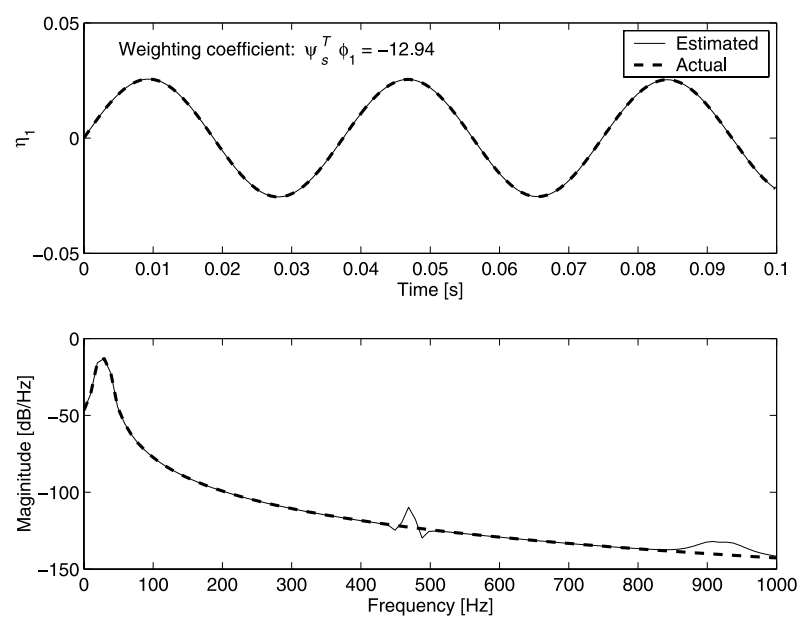

Fig. 10 Filtered response for E-set sensor placement (modal filtering of the first mode)

\section{Acknowledgement}

The authors would like to thank the SUZUKI foundation for financial support.

\section{References}

( 1 ) Liu, W. and Hou, Z., Model Reduction in Structural Vibration Control and Its Application, 16th ASCE Engineering Mechanics Conference, (2003).

( 2 ) Balas, M. J., Active Control of Flexible Structures, Journal of Optimization Theory and Applications, Vol. 25, No. 3, (1978), pp. 415-437.

( 3 ) Meirovitch, L. and Baruh, H., Control of Self-Adjoint Distributed Parameter Systems, Journal of Guidance, Control and Dynamics, Vol. 5, No. 1, (1982), pp. 60-66.

( 4 ) Shelly, S. J., Freudinger, L. C. and Allemang, R. J., Development of an On-Line Parameter Estimation System Using the Discrete Modal Filter, Proceedings of 10th International Modal Analysis Conference, (1992), pp. 173-183.

( 5 ) Tanaka, N., et al., Spatial Modal Filtering Using Point Sensors, Transactions of the Japan Society of Mechanical Engineers, Series C, Vol.63, No.609 (1997), pp. 15591567.

( 6 ) Matsumura, Y., Uncertainty in Cross Orthogonality Checks, JSME International Journal, Series C, Vol. 46, No. 2, (2003), pp. 692-698.

( 7 ) Doebling, S. W., Farrar, C. R., Prime, M. B. and Shevitz, D. W., Damage Identification and Health Monitoring of Structural and Mechanical Systems form Changes in Their Vibration Characteristics: A Literature Review, Los Alamos National Laboratory Technical Report, LA-13070-MS, (1996). 